\title{
Central Sleep Apnea without Cheyne-Stokes Breathing Following an Acute Cerebral Infarction
}

\author{
Hye Jeong $\mathrm{Oh}^{1}$, Ho Geol Woo ${ }^{2}$, Jin Myoung Seok ${ }^{1}$, Kwang Ik Yang ${ }^{1}$ \\ ${ }^{1}$ Sleep Disorders Center, Department of Neurology, Soonchunhyang University College of Medicine, Cheonan Hospital, Cheonan, \\ ${ }^{2}$ Department of Neurology, Ewha Womans University College of Medicine, Seoul, Korea
}

\author{
Received February 8, 2019 \\ Revised May 1, 2019 \\ Accepted May 22, 2019 \\ Address for correspondence \\ Kwang Ik Yang, MD, PhD \\ Sleep Disorders Center, \\ Department of Neurology, \\ Soonchunhyang University \\ College of Medicine \\ Cheonan Hospital, \\ 31 Suncheonhyang 6-gil, \\ Dongnam-gu, Cheonan 31151, \\ Korea \\ Tel: $+82-41-570-2290$ \\ Fax: +82-41-592-3810 \\ E-mail: neurofan@schmc.ac.kr
}

\begin{abstract}
Central sleep apnea (CSA) is attributed to medical or neurological conditions including stroke. The association of lesion location and CSA in patients with ischemic stroke has not been well elucidated. A 69-year-old man with a history of hypertension and diabetes mellitus was admitted due to stroke. The brain magnetic resonance imaging showed an acute ischemic stroke in the right ventral thalamus and adjacent hypothalamus. During hospitalization, polysomnography (PSG) was performed because repetitive cessation of respiration during sleep was observed by chance. PSG showed severe CSA; the apnea-hypopnea index (AHI) was 73.5 with a minimum oxygen saturation of $89 \%$ and central apnea index (CAI) was 63.0. Two years later, follow-up PSG showed that AHI was 7.2 with a minimum oxygen saturation of $91 \%$ and CAI was 1.0. We report the patient with CSA after ischemic stroke with right thalamus and adjacent hypothalamus, which resolved spontaneously with time.

J Sleep Med 2019;16(1):53-55
\end{abstract}

Sleep related breathing disorders (SRBDs) are common in patients with stroke. ${ }^{1}$ However, the relationship between SRBD and stroke is not clear. Central sleep apnea (CSA) is characterized by a loss of central respiratory drive and effort during sleep, resulting in insufficient or absent ventilation, rather than physical upper-airway collapse. ${ }^{2}$ CSA is attributed to medical or neurological conditions including stroke. Although brainstem infarctions have previously been suggested to be associated with CSA, ${ }^{3,4}$ the association of lesion location and CSA in patients with ischemic stroke has not been well elucidated. We report a patient with CSA after acute cerebral infarction.

\section{Case Report}

A 69-year-old man with a history of hypertension and diabetes mellitus was admitted to hospital complaining of left hemiparesis, facial palsy, and dysarthria one day prior to admission. Initial National Institutes of Health Stroke Scale (NI-

This is an Open Access article distributed under the terms of the Creative Commons Attribution Non-Commercial License (https://creativecommons.org/licenses/by-nc/4.0) which permits unrestricted non-commercial use, distribution, and reproduction in any medium, provided the original work is properly cited.
HSS) was 7. Magnetic resonance imaging revealed an acute infarction in the right ventral thalamus and adjacent hypothalamus (Fig. 1).

Electrocardiogram, transthoracic echocardiography, transesophageal echocardiography, 24-hours Holter monitoring, and chest X-ray were unremarkable. There was no other history of medical issues and drug use. During hospitalization, repetitive cessation of respiration during sleep was observed by chance. But there was no remarkable finding of respiration when he was wakefulness. He had no suggestive symptoms of snoring and sleep apnea before the stroke. Polysomnography (PSG) (COMET PSG; Twin 4.5.2 Software, Grass Technologies, Warwick, RI, USA) was performed, which showed severe CSA with 324 respiratory events consisting of 295 central apneas, and 29 obstructive hypopneas. The events of CSA were not associated with Cheyne-Stokes breathing (CSB). The overall apnea-hypopnea index (AHI) was 73.5 with a minimum oxygen saturation of $89 \%$. Central apnea index (CAI) was 63.0 (Fig. 2). The respiratory events were not associated with significant oxygen desaturation below $88 \%$. So, nocturnal non-invasive ventilation such as adaptive servo-ventilation was not applied. Two years later, the patient had no sequelae of 


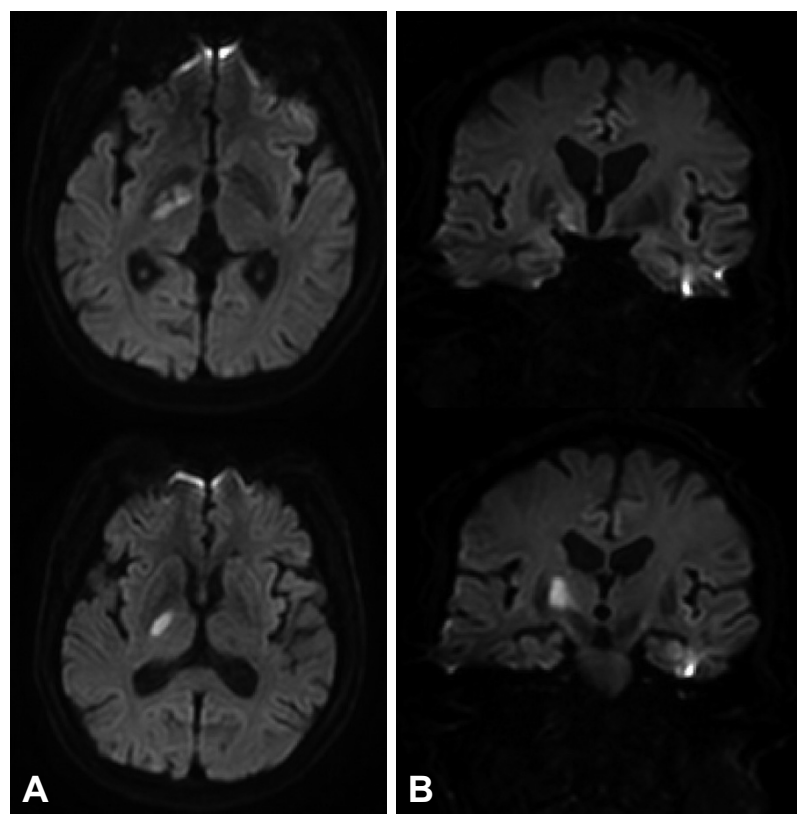

Figure 1. Diffusion weighted imaging of the patient. Axial view (A) and coronal view (B) shows acute ischemic stroke in the right ventral thalamus and adjacent hypothalamus. stroke and follow up PSG showed that AHI was 7.2 with a minimum oxygen saturation of $91 \%$ and CAI was 1.0 (Table 1).

\section{Discussion}

Although obstructive apnea is more common, central apnea may be noted initially after stroke. It is difficult to determine the relationship between prior and post-stroke SRBD in case of no prior objective diagnosis of SRBD. ${ }^{2}$ Although our case did not have PSG finding before stroke onset, it is reasonable to assume that CSA caused by acute ischemic stroke because it resolved spontaneously over two years. The CSA following a stroke is abrupt in onset and tend to resolve with time. ${ }^{5}$ However, well-illustrated PSG finding showing typical CSA without CSB has rarely been reported. On this case, the CAI was normalized and markedly reduced compared to initial PSG (63.0 vs. 1.0).

This case showed acute ischemic lesion in the right ventral thalamus and adjacent hypothalamus. Post-stroke sleep-disordered breathing has been well introduced but no specific lesion location was related to the SRBD as well as CSA. ${ }^{6}$ Previous studies showed that brainstem stroke caused $\mathrm{CSB}^{4}$ and

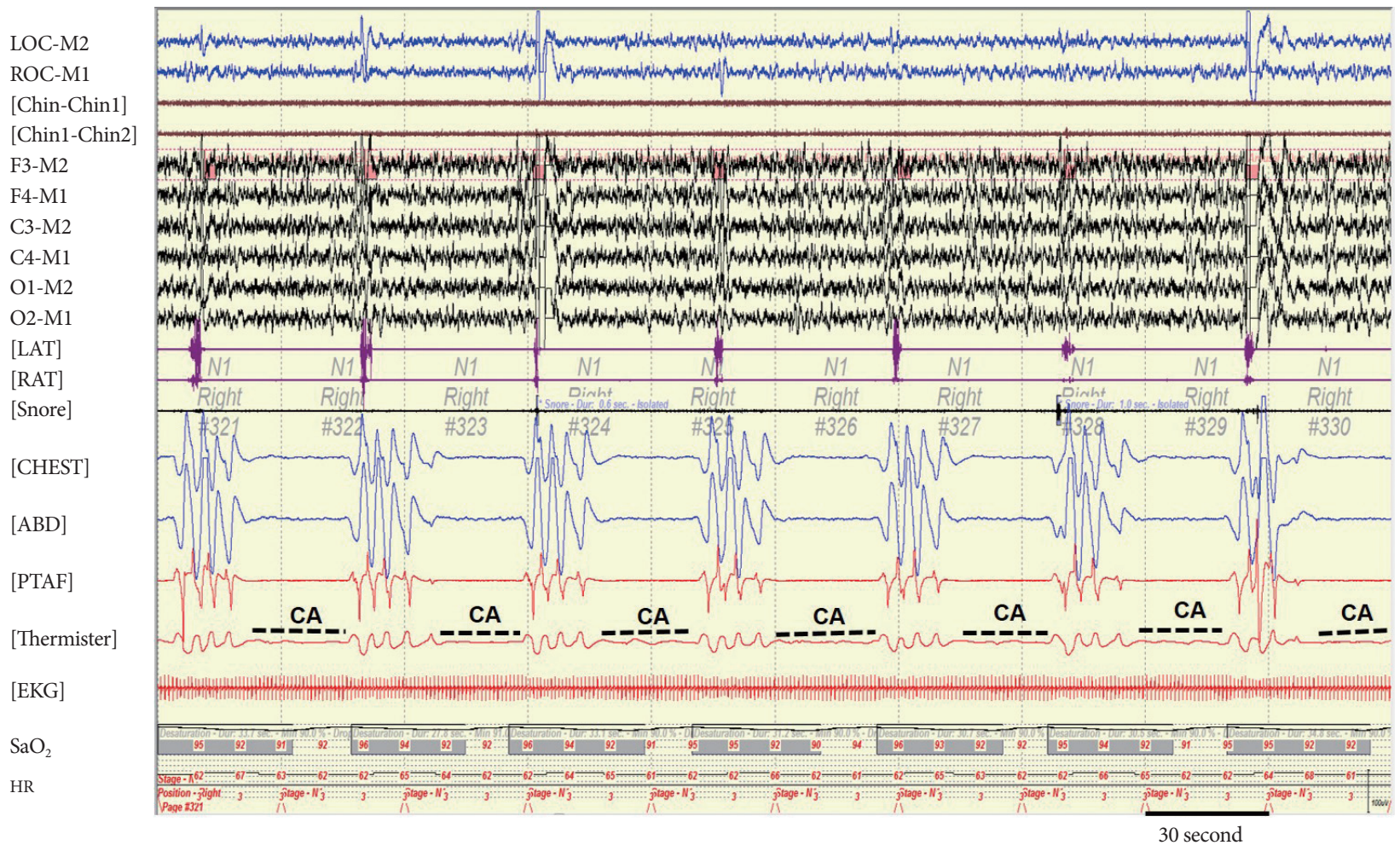

Figure 2. Polysomnographic (PSG) finding 3 days after stroke. A 5-minutes epoch PSG shows periodic central respiratory events without Cheyne-Stokes breathing. The overall apnea-hypopnea index was 73.5 and central apnea index was 63.0. Two electrooculogram channels (LOC-M2, ROC-M1), two chin electromyogram channels (Chin-Chin 1, Chin1-Chin 2), and six electroencephalogram channels (F3-M2, F4M1, C3-M2, C4-M1, O1-M2, O2-M1) were measured. LAT: left anterior tibialis electromyogram, RAT: right anterior tibialis electromyogram, Snore: snore sensor, CHEST: chest respiratory movement, ABD: abdomen respiratory movement, PTAF: pressure transducer air-flow sensor, Thermister: oro-nasal thermal sensor, EKG: electrocardiogram, $\mathrm{SaO}_{2}$ : oxyhemoglobin saturation by pulse oximetry, HR: heart rate, CA: central apnea. 
Table 1. Polysomnographic parameters between initial and followup study

\begin{tabular}{lcc}
\hline & Initial PSG & PSG after 2 years \\
\hline Total recording time, min & 496.5 & 441 \\
Total sleep time, min & 264.5 & 301.5 \\
Total stage N1, min & $121(24.4 \%)$ & $55.5(12.6 \%)$ \\
Total stage N2, min & $143.5(28.9 \%)$ & $195.5(44.3 \%)$ \\
Total stage N3, min & 0 & 0 \\
Total stage R, min & 0 & $50.5(11.5 \%)$ \\
Wake after sleep onset, min & 230.5 & 119.5 \\
Latency to sleep onset, min & 1.5 & 20 \\
Latency to REM sleep, min & $\mathrm{NC}$ & 102 \\
Sleep efficiency, min & $53.3 \%$ & $68.4 \%$ \\
Apnea-hypopnea index & 68.7 & 7.2 \\
Central apnea index & 63.0 & 1.0 \\
Nadir oxygen saturation & $89 \%$ & $91 \%$ \\
\hline
\end{tabular}

PSG: polysomnography, REM: rapid eye movement, NC: no contribution

high prevalence of CSA without CSB. ${ }^{3}$ In a few additional reports, various lesions such as cortical and subcortical area have been reported to be associated with central periodic breathing during sleep in acute ischemic stroke. The mechanism of CSA after stroke has been discussed to be a direct consequence of the injury of central nervous system structures, which is involving autonomic and volitional respiratory centers. ${ }^{8,9}$ Within the brainstem, the medulla oblongata contains central chemoreceptors that response to a loop-gain feedback system on the $\mathrm{PaCO}_{2}{ }^{10}$ Lesions to this area lead to decrease chemosensitivity during sleep and develop CSA. Besides, non-brainstem regions might have role of autonomic and voluntary respiration involving insular, and supplementary motor cortex and thalamus. ${ }^{8,9}$ It is also known that some hypothalamic nuclei are interconnected with respiratory nuclei located in the brainstem and involve respiratory control as well as other autonomic brain function. Although dysfunction of hypothalamus causes abnormal breathing, the role of the hypothalamus in regulation of respiration during sleep remain to be elucidated. ${ }^{11}$ Similarly, it is not well known yet about specific anatomical structures and pathomechanism related to CSA after stroke.

This case demonstrates an example of CSA without CSB after ischemic stroke with right thalamus and adjacent hypo- thalamus, which resolved spontaneously with time. Further elucidation of mechanisms how these structures regulates involuntary respiratory function during sleep is required.

\section{Conflicts of Interest}

The authors have no potential conflicts of interest to disclose.

\section{ORCID iDs}

$\begin{array}{ll}\text { Hye Jeong Oh } & \text { https://orcid.org/0000-0002-3622-9945 } \\ \text { Ho Geol Woo } & \text { https://orcid.org/0000-0001-6489-0100 } \\ \text { Jin Myoung Seok } & \text { https://orcid.org/0000-0002-1484-2968 } \\ \text { Kwang Ik Yang } & \text { https://orcid.org/0000-0001-6343-6520 }\end{array}$

\section{Author Contributions}

Conceptualization: Hye Jeong Oh, Ho Geol Woo, Jin Myoung Seok, Kwang Ik Yang. Data curation: Hye Jeong Oh, Kwang Ik Yang. Formal analysis: Hye Jeong Oh, Kwang Ik Yang. Investigation: Hye Jeong Oh, Ho Geol Woo, Jin Myoung Seok, Kwang Ik Yang. Methodology: Hye Jeong Oh, Ho Geol Woo, Jin Myoung Seok, Kwang Ik Yang. Supervision: Kwang Ik Yang. Writing_-original draf: Hye Jeong Oh, Kwang Ik Yang. Writingreview \& editing: Hye Jeong Oh, Kwang Ik Yang.

\section{REFERENCES}

1. Johnson KG, Johnson DC. Frequency of sleep apnea in stroke and TIA patients: a meta-analysis. J Clin Sleep Med 2010;6:131-137.

2. Stevens D, Martins RT, Mukherjee S, Vakulin A. Post-stroke sleep-disordered breathing-pathophysiology and therapy options. Front Surg 2018;5:9.

3. Lee MC, Klassen AC, Heaney LM, Resch JA. Respiratory rate and pattern disturbances in acute brain stem infarction. Stroke 1976;7:382-385.

4. Nachtmann A, Siebler M, Rose G, Sitzer M, Steinmetz H. CheyneStokes respiration in ischemic stroke. Neurology 1995;45:820-821.

5. American Academy of Sleep Medicine. International classification of sleep disorders. 3rd ed. Darien: American Academy of Sleep Medicine, 2014.

6. Fisse AL, Kemmling A, Teuber A, et al. The association of lesion location and sleep related breathing disorder in patients with acute ischemic stroke. PLoS One 2017;12:e0171243.

7. Hermann DM, Siccoli M, Kirov P, Gugger M, Bassetti CL. Central periodic breathing during sleep in acute ischemic stroke. Stroke 2007;38: 1082-1084.

8. Aleksandrov VG, Aleksandrova NP, Bagaev VA. Identification of a respiratory related area in the rat insular cortex. Can J Physiol Pharmacol 2000;78:582-586.

9. Thornton JM, Guz A, Murphy K, et al. Identification of higher brain centres that may encode the cardiorespiratory response to exercise in humans. J Physiol 2001;533(Pt 3):823-836.

10. Eckert DJ, Jordan AS, Merchia P, Malhotra A. Central sleep apnea: pathophysiology and treatment. Chest 2007;131:595-607.

11. Fukushi I, Yokota S, Okada Y. The role of the hypothalamus in modulation of respiration. Respir Physiol Neurobiol 2019;265:172-179. 